\title{
Association between peripheral manganese levels and attention-deficit/hyperactivity disorder: a preliminary meta-analysis
}

This article was published in the following Dove Press journal:

Neuropsychiatric Disease and Treatment

Jun-Hao Shih, , 2,* Bing-Yan Zeng, ${ }^{3, *}$ Pao-Yen Lin, ${ }^{4,5}$ Tien-Yu Chen, ${ }^{6,7}$ Yen-Wen Chen, ${ }^{8}$ Ching-Kuan Wu, ${ }^{9}$ Ping-Tao Tseng, ${ }^{10}$ Ming-Kung $\mathrm{Wu}^{4}$

'Department of Occupational and Environmental Health Consultation, EMHA Consulting International Incorporation, Hsinchu, Taiwan; ${ }^{2}$ Taiwan Environmental and Occupational Medicine Association,

Tainan, Taiwan; ${ }^{3}$ Department of Internal Medicine, E-Da Hospital, Kaohsiung, Taiwan; ${ }^{4}$ Department of Psychiatry, Kaohsiung Chang Gung Memorial Hospital and Chang Gung University College of Medicine, Kaohsiung, Taiwan; ${ }^{5}$ Center for Translational Research in Biomedical Sciences, Kaohsiung Chang Gung Memorial Hospital and Chang Gung University College of Medicine, Kaohsiung, Taiwan; ${ }^{6}$ Department of Psychiatry, Tri-Service General Hospital, School of Medicine, National Defense Medical Center, Taipei, Taiwan; ${ }^{7}$ Institute of Brain Science, National Yang-Ming University, Taipei, Taiwan; ${ }^{8}$ Prospect Clinic for Otorhinolaryngology and Neurology, Kaohsiung, Taiwan; ${ }^{9}$ Department of Psychiatry, Tsyr-Huey Mental Hospital, Kaohsiung Jen-Ai's Home, Kaohsiung, Taiwan; ${ }^{10}$ WinShine Clinics in Specialty of Psychiatry, Kaohsiung, Taiwan

*These authors contributed equally to this work

Correspondence: Ping-Tao Tseng WinShine Clinics in Specialty of Psychiatry, No 68, Nantai Road, Xinxing District, Kaohsiung 800, Taiwan

Tel/fax +88672850119

Email ducktseng@gmail.com

Ming-Kung Wu

Department of Psychiatry, Kaohsiung Chang Gung Memorial Hospital and Chang Gung University College of Medicine, No 123, Dapi Road, Niaosong District, Kaohsiung 833, Taiwan

Tel +886 7 73। 7II23 ext 8753

Fax +8867732 68I7

Email mingkungl80@gmail.com

\begin{abstract}
Evidence has suggested that dysregulation of the dopaminergic system may play a significant role in the pathogenesis of attention-deficit/hyperactivity disorder (ADHD) in children. Manganese, a neurotoxicant, has been reported to exert its neurotoxicity by affecting the dopaminergic system. However, the association between peripheral manganese levels and ADHD has not been comprehensively reviewed. This study aimed to investigate the association between peripheral manganese levels and ADHD in children. An electronic search was performed on databases including PubMed, ProQuest, ClinicalKey, Cochrane Library, ClinicalTrials.gov, Embase, Web of Science, and ScienceDirect with last search on March 25th, 2018. As per the inclusion criteria, human observational studies investigating peripheral manganese levels in children with ADHD and controls were included. The meta-analysis was performed using a random-effects model, and possible confounders were examined by subgroup analysis. In total, four articles with 175 ADHD children and 999 controls were recruited. The manganese levels were significantly higher in ADHD children than in controls $(p=0.033)$, when studies investigating blood levels and those investigating hair levels were included. However, when only studies investigating blood levels were included, there was no significant difference between ADHD children and controls $(p=0.076)$. Our results support higher peripheral manganese levels in children diagnosed with ADHD than those in controls. Further primary studies are needed to clarify this association.
\end{abstract}

Keywords: meta-analysis, manganese, ADHD, pediatric psychiatry, review

\section{Introduction}

Attention-deficit/hyperactivity disorder (ADHD) is defined as "a persistent pattern of inattention and/or hyperactivity-impulsivity that interferes with functioning or development". ${ }^{1}$ ADHD can be categorized into three subtypes: predominantly inattentive, predominantly hyperactive-impulsive, and combined. ${ }^{2}$ The prevalence of ADHD is estimated to be $5.29 \%$ in children worldwide. ${ }^{3}$ Inattention refers to wandering off task, lacking persistence, having difficulty sustaining focus, and being disorganized and is not due to defiance or lack of comprehension. Impulsivity refers to excessive motor activity when it is not appropriate, or excessive fidgeting, tapping, or talkativeness. ${ }^{1}$ Further, it may cause impairments in social communication and functional limitations of effective communication, social participation, or academic achievement. ${ }^{1}$

Although the exact pathogenesis of ADHD remains unknown, it has been found that people with ADHD have smaller brain volumes of specific structures, such as the prefrontal cortex and basal ganglia, ${ }^{2}$ or have higher exposure to environmental pollution, such as lead ${ }^{4}$ or polybrominated diphenyl ethers. ${ }^{5}$ One of the most widely submit your manuscript Dovepress fy http://dx.doi.org/10.2147/NDT.S|65378 cc. hereby accept the Terms. Non-commercial uses of the work are permitted without any further permission from Dove Medical Press Limited, provided the work is properly attributed. For permission for commercial use of this work, please see paragraphs 4.2 and 5 of our Terms (https://www.dovepress.com/terms.php). 
postulated pathogeneses of ADHD is the "dopamine hypothesis", ${ }^{2}$ attributing ADHD to a dysregulation of the dopaminergic system. A review article has shown that ADHD patients have decreased availability of dopamine receptor isoforms and increased dopamine transporter (DAT) binding compared with controls. ${ }^{6}$ The most widely used medication for ADHD, methylphenidate with its derivatives, is postulated to have its effects by blocking DAT and promoting dopamine release. ${ }^{6}$

In previous studies, manganese (elemental symbol: Mn), an environmental heavy metal element, has been found to cause neurologic toxicity to humans. ${ }^{7,8}$ It can be present in contaminated water, pesticides, batteries, and glass, among others, and then be ingested by humans. ${ }^{9-11}$ High concentration of manganese has been found in the basal ganglia of manganese-intoxicated rats. ${ }^{12}$ After exposure to manganese, decreased long-term dopamine efflux in rats ${ }^{13}$ and attenuated dopaminergic neurotransmission in humans ${ }^{14}$ have also been reported. In epidemiological studies, higher levels of manganese in the hair and blood samples from children with ADHD have been found. ${ }^{15,16}$ Another study has shown decreased intelligence quotient (IQ) in children with increased hair concentration of manganese. ${ }^{17}$ There have been no studies investigating the postmortem levels of in vivo manganese in ADHD patients found in the literature, by using the same search strategy as the current meta-analysis.

Therefore, as a neurotoxin affecting dopaminergic neurotransmission, manganese may play a role in the pathogenesis of ADHD in those exposed to the metal. Among the four datasets in the literature studying "blood" manganese levels, two ${ }^{16,18}$ showed a significantly positive association between blood manganese level and ADHD, while the other two ${ }^{18,19}$ revealed insignificant results. Three of the four datasets used case-control design, while the other dataset used cross-sectional design. Another case-control study showed a trend of positive association between "hair" manganese level and ADHD without statistical significance. ${ }^{20}$ Difference in study design (case-control vs cross-sectional), source of manganese level (ie, blood vs hair), and population (described in the "Discussion" section) may contribute to the heterogeneity of the study results. Although there are two previous meta-analyses ${ }^{17,21}$ mentioning the possible relationship between manganese exposure and ADHD in children, they did not directly calculate the association. Therefore, the aim of the current study is to summarize the literature for the possible association between peripheral manganese levels and ADHD in children via a thorough meta-analysis.

\section{Methods}

We followed the Meta-analysis Of Observational Studies in Epidemiology (MOOSE) guidelines ${ }^{22}$ (Figure S1). This preliminary meta-analysis followed an a priori defined but unpublished protocol. The Institutional Review Board of the Tri-Service General Hospital (TSGHIRB: B-105-12) approved the study.

\section{Eligibility criteria}

Inclusion criteria were the following: (a) observational studies, including a cohort or cross-sectional study design comparing peripheral levels of manganese in children with ADHD and controls; and (b) trials in humans. We excluded preclinical studies, review articles, meeting abstracts, and non-human studies; furthermore, to increase the specificity of the effect of manganese on ADHD, we excluded those studies that collected manganese sample sources further away from the diagnosis of ADHD (eg, collecting manganese when ADHD children were just delivered).

\section{Search strategy and study selection}

Two well-trained authors (JH Shih and PT Tseng) performed a systematic literature search from inception until August 30th, 2017 and updated search at March 25th, 2018. The following keywords were used in our search on PubMed, ProQuest, ClinicalKey, Cochrane Library, ScienceDirect, and ClinicalTrials.gov platforms: "(Manganese OR Mn) AND (ADHD OR attention-deficit/hyperactivity disorder)". A similar search was conducted in Embase and Web of Science databases. The reference lists of included articles and recent reviews were also hand-searched to identify additional articles. ${ }^{17,21,23-25}$

Two authors (JH Shih and PT Tseng) independently screened the titles and abstracts of all retrieved results for potential eligibility. Both authors reviewed the full text of potentially eligible papers, and a final list of included studies was achieved. Any inconsistencies were resolved through discussion with a third reviewer (MK Wu).

\section{Data extraction}

The primary outcomes were the difference in manganese levels in children with ADHD compared to control groups (calculated as Hedges' $g$ statistic and corresponding 95\% CIs and $p$-values). We did not choose difference in mean as the effect sizes (ESs) of our primary outcome due to presumed different units used in each study. The control groups were defined as those without ADHD. In addition, 
due to potential confounding effect of specific medication (eg, methylphenidate) on manganese, ${ }^{18}$ we did not include the data of manganese level in children with ADHD who had been clearly described as receiving such medication. If data were available, we prefer to choose control groups as healthy (asymptomatic) individuals. When data were not available from the included studies, we contacted the primary authors to request the original data. We contacted the authors via email on two occasions if required (a second email was sent a week later if no response was received following an initial email). If there were no relevant data in the paper regarding manganese levels, wherever possible, we attempted to use another compatible statistical parameter (eg, $p$-value and sample sizes) to estimate the ESs according to the protocol in the Comprehensive Meta-Analysis manuals and guidance on the Comprehensive Meta-Analysis website (https://www. meta-analysis.com/downloads/Meta-analysis $\% 20$ Converting $\% 20$ among $\% 20$ effect $\% 20$ sizes.pdf) to covert and pool the ESs into Hedges' $g$.

The variables of interest included manganese levels, mean age, gender distribution (in the form of $\%$ females), mean body mass index, cognitive performance (in the form of mean IQ), parental tobacco smoking and alcohol consumption, and the type of assay used for detection of manganese levels.

\section{Methodological quality appraisal}

We used the modified Newcastle-Ottawa Scale (NOS) to evaluate the quality of the included studies. In brief, the modified NOS was based on a version previously used in a meta-analysis study published in British Journal of Psychiatry in 2013, and score ranges from 0 to 6 (Table S1). ${ }^{26}$

\section{Meta-analysis procedure}

Given the anticipated heterogeneity in the basic population of study, we conducted the meta-analyses with a random-effects model rather than a fixed-effects one. ${ }^{27}$ As aforementioned, the primary ES was estimated as Hedges' $g$ with 95\% CI to compare manganese levels in children with ADHD and controls. Subgroup analyses were conducted to determine whether results differed as we grouped them according to the sample sources (eg, blood, hair). We performed subgroup analyses whenever data from at least two independent datasets were available. In addition, to overcome the limitation of the meta-analysis that we could not adjust the potential confounding effect by clinical variables, we collected the adjusted OR provided by the recruited articles to calculate the pooled adjusted OR.

\section{Sensitivity test, heterogeneity, publication bias, and meta-regression}

We performed sensitivity test with "one-study removal" test to evaluate if the results of the meta-analysis were obtained from any outliers within the recruited studies or not. ${ }^{28}$ Heterogeneity was assessed by the Cochran $Q$ test and its corresponding $p$-value. ${ }^{29}$ The $I^{2}$ statistic was interpreted as the proportion of heterogeneity a study estimates that is due to heterogeneity. ${ }^{30} \mathrm{We}$ performed meta-regression analyses with unrestricted maximum likelihood random effects when data on each potential moderator were provided by at least five different studies. We assessed publication bias via the inspection of funnel plots ${ }^{31}$ and with the Egger's regression test. $^{32}$ When evidence of publication bias was found, the Duval and Tweedie's trim-and-fill procedure, which is a validated model to estimate an ES, was employed. ${ }^{33}$ The current meta-analysis was conducted using the Comprehensive Meta-Analysis software, version 3 (BioStat, Frederick, MD, USA). The threshold for statistical significance was set at a two-tailed $p$-value $<0.05$.

\section{Results Study selection}

Figure 1 summarizes study selection process. In brief, a total of 22 studies entered the full-text review stage. Eighteen articles were excluded for various reasons (Figure 1). A list of excluded articles is presented in Table S2. In total, four articles met the inclusion criteria, the details of which are summarized in Table $1 .^{16,18-20}$

Among the included studies, four provided comparison of the peripheral manganese levels in children with ADHD and controls (children with ADHD: $\mathrm{n}=175$, mean age $=8.8$, mean female proportion $=16.0 \%$; controls without ADHD: $n=999$, mean age $=9.1$, mean female proportion $=45.9 \%) \cdot{ }^{16,18-20}$ Two of them provided the data of adjusted OR for the association between manganese levels and ADHD. ${ }^{16,20}$

\section{Characteristics and methodological quality of included studies}

Among the recruited studies, the diagnosis of ADHD was made according to widely acceptable criteria, including the Diagnostic and Statistical Manual of Mental Disorders $(\mathrm{DSM})^{16,18,20}$ or Diagnostic Interview Schedule for Children Version IV. ${ }^{19}$ In addition, the sample sources ranged from peripheral blood ${ }^{16,18,19}$ to hair sample. ${ }^{20}$ The assays used to detect manganese levels included inductively coupled plasma mass spectrometry and graphite furnace atomic absorption spectrometry (Table S1). Regarding the methodological 


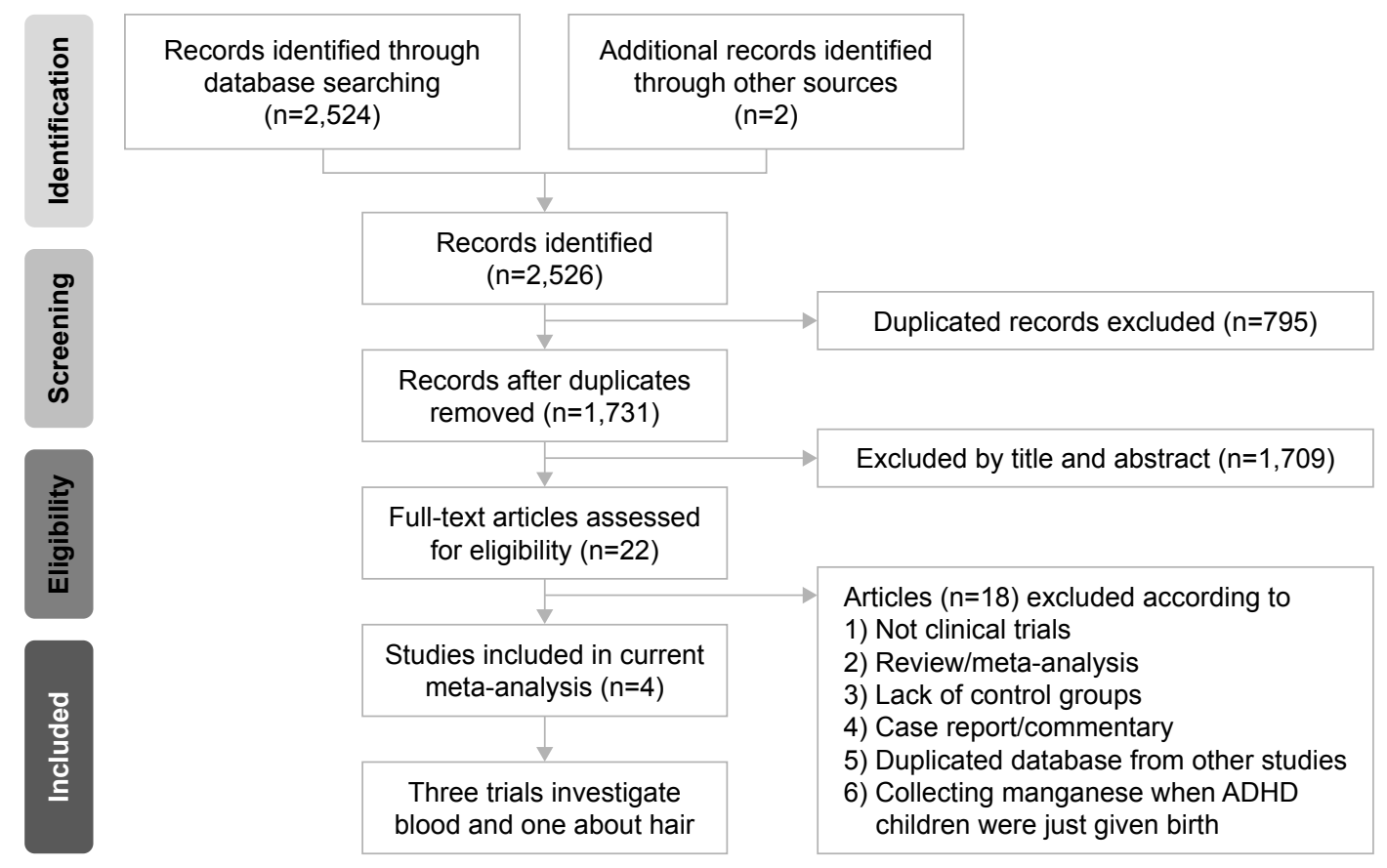

Figure I The flowchart of meta-analysis.

Abbreviation: ADHD, attention-deficit hyperactivity disorder.

quality of included studies, the average NOS score was 15.5 with a SD of 1.0 (Table S1).

\section{Meta-analysis of overall differences in manganese levels in children with ADHD and controls without ADHD Overall}

Four articles provided five datasets in this part of metaanalysis. ${ }^{16,18-20}$ The meta-analysis suggested that overall manganese levels, either in blood or in hair, in children diagnosed with ADHD were significantly higher than those in controls ( $k=5$, Hedges' $g=0.305,95 \% \mathrm{CI}=0.025-0.585$, $p=0.033$ ) (Figure 2A) without significant evidence of heterogeneity $\left(Q\right.$ value $=8.419, d f=4, I^{2}=52.486 \%, p=0.077$, tau $=0.229)$, but significant publication bias via inspection of funnel plot and Egger's regression was found ( $t=10.525$, $d f=3, p=0.002$ ). The significant results of the meta-analysis changed to insignificant after refilling two potential missing data to the left side of means according to Duval and Tweedie's trim-and-fill test (Hedges' $g=0.145,95 \%$ $\mathrm{CI}=-0.140$ to 0.430 ).

\section{Sensitivity test}

The significant results of the meta-analysis changed to insignificant after removing any one of the recruited studies, which might be due to smaller sample sizes after removal of one of the recruited studies. However, the significant results of the meta-analysis changed to more predominantly significant after removing the data from Hong et al (Hedges' $g=0.425$, $95 \% \mathrm{CI}=0.191-0.660, p<0.001){ }^{19}$

\section{Meta-regression}

The meta-regression could be performed only on association between ESs and mean age due to lack of data. To be specific, there was no significant association between ESs and mean age $(p=0.656)$.

\section{Meta-analysis of differences in manganese levels in children with ADHD and controls without ADHD in blood samples Blood manganese}

Three articles provided four datasets in this part of metaanalysis. ${ }^{16,18,19}$ The meta-analysis suggested that the blood manganese levels in children diagnosed with ADHD and controls without ADHD were not significantly different, but a trend of higher blood manganese levels in ADHD children compared to controls was observed ( $k=4$, Hedges' $g=0.329,95 \% \mathrm{CI}=-0.035$ to $0.693, p=0.076$ ) (Figure $2 \mathrm{~B}$ ) with significant evidence of heterogeneity $(Q$ value $=8.394$, $d f=3, I^{2}=64.260 \%, p=0.039$, tau $\left.=0.295\right)$ and significant publication bias via inspection of funnel plot and Egger's regression $(t=9.335, d f=2, p=0.011)$. However, the results 


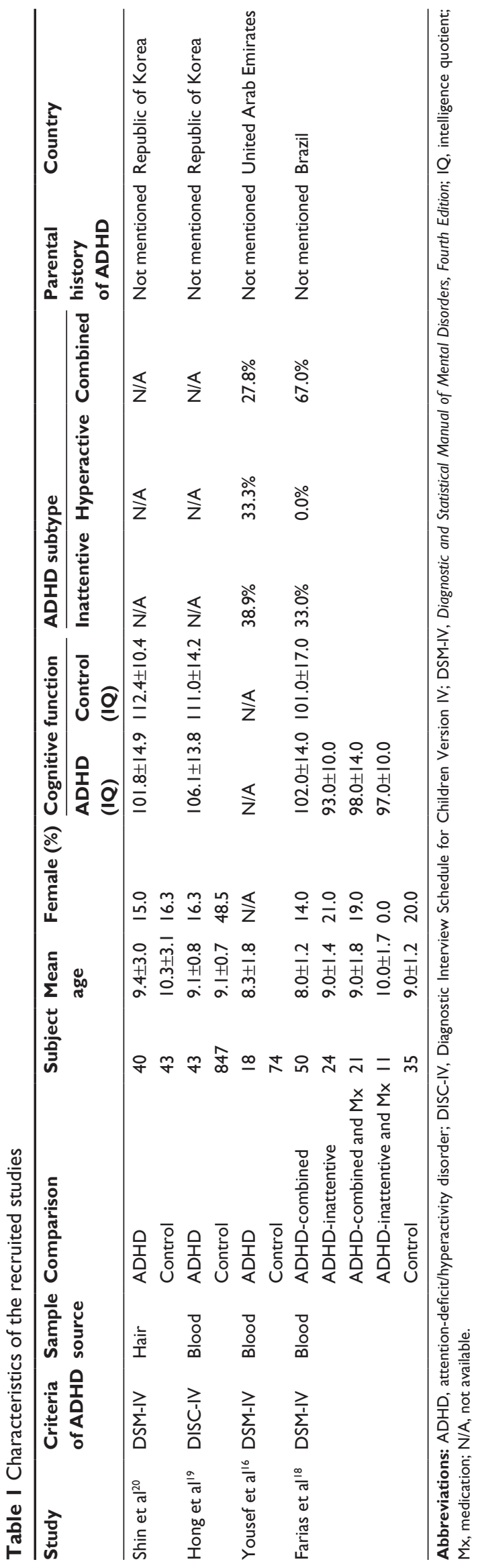

of the meta-analysis did not change after refilling according to Duval and Tweedie's trim-and-fill test.

\section{Sensitivity test}

The insignificant results of the meta-analysis changed to significant after removing the data from Hong et al (Hedges' $g=0.491,95 \%$ CI $=0.210-0.771, p=0.001)$.

\section{Meta-regression}

All the meta-regression procedures were not performed because there were less than five datasets.

\section{Meta-analysis of differences in manganese levels in children with ADHD and controls without ADHD in hair samples Hair manganese}

Only one article provided one dataset in this part of metaanalysis. ${ }^{20}$ Therefore, the meta-analysis was not done due to lack of enough datasets. However, in the research results according to the description in the article by Shin et al, ${ }^{20}$ the authors indicated that the proportion of abnormal manganese levels, either higher or lower, in children with ADHD was significantly higher than those in controls $(p=0.039)$.

\section{Meta-analysis of overall adjusted OR for the association between manganese levels and diagnosis of ADHD}

Two articles provided two datasets in this part of metaanalysis. ${ }^{16,20}$ The meta-analysis of the adjusted ORs indicated significant association between manganese levels and the diagnosis of ADHD ( $k=2$, adjusted OR=1.849, 95\% CI=1.149-2.976, $p=0.011$ ) (Figure 2C) without significant evidence of heterogeneity ( $Q$ value $=0.150, d f=1$, $I^{2}<0.001 \%, p=0.698$, tau $\left.<0.001\right)$.

\section{Discussion}

To the best of our knowledge, this is the first meta-analysis to investigate the relationship between peripheral manganese levels and ADHD in children. The results of the current metaanalysis showed significantly higher overall (ie, in blood or hair) peripheral manganese levels in children diagnosed with ADHD than those in controls, with no significant heterogeneity. Among the four included studies with five datasets in total, our results are consistent with two datasets, ${ }^{16,18}$ but not with the other three. ${ }^{18-20}$ However, among the three studies mentioned, ${ }^{18-20}$ two ${ }^{18,20}$ also showed a trend of positive association ( $p=0.146$ and 0.213 , respectively), though 
not significant, which may be due to small number of cases. Sensitivity test showed that the results became insignificant after removing any one of the included studies, which may also be due to small number of cases. Subgroup analysis showed a trend of higher "blood" manganese levels in ADHD children than in controls, though not significant $(p=0.076)$. However, the results changed to significant after removing the data from Hong et al. ${ }^{19}$ The change from insignificance to significance may have been due to the difference in the study designs. Among the four studies included in the current

\section{A}

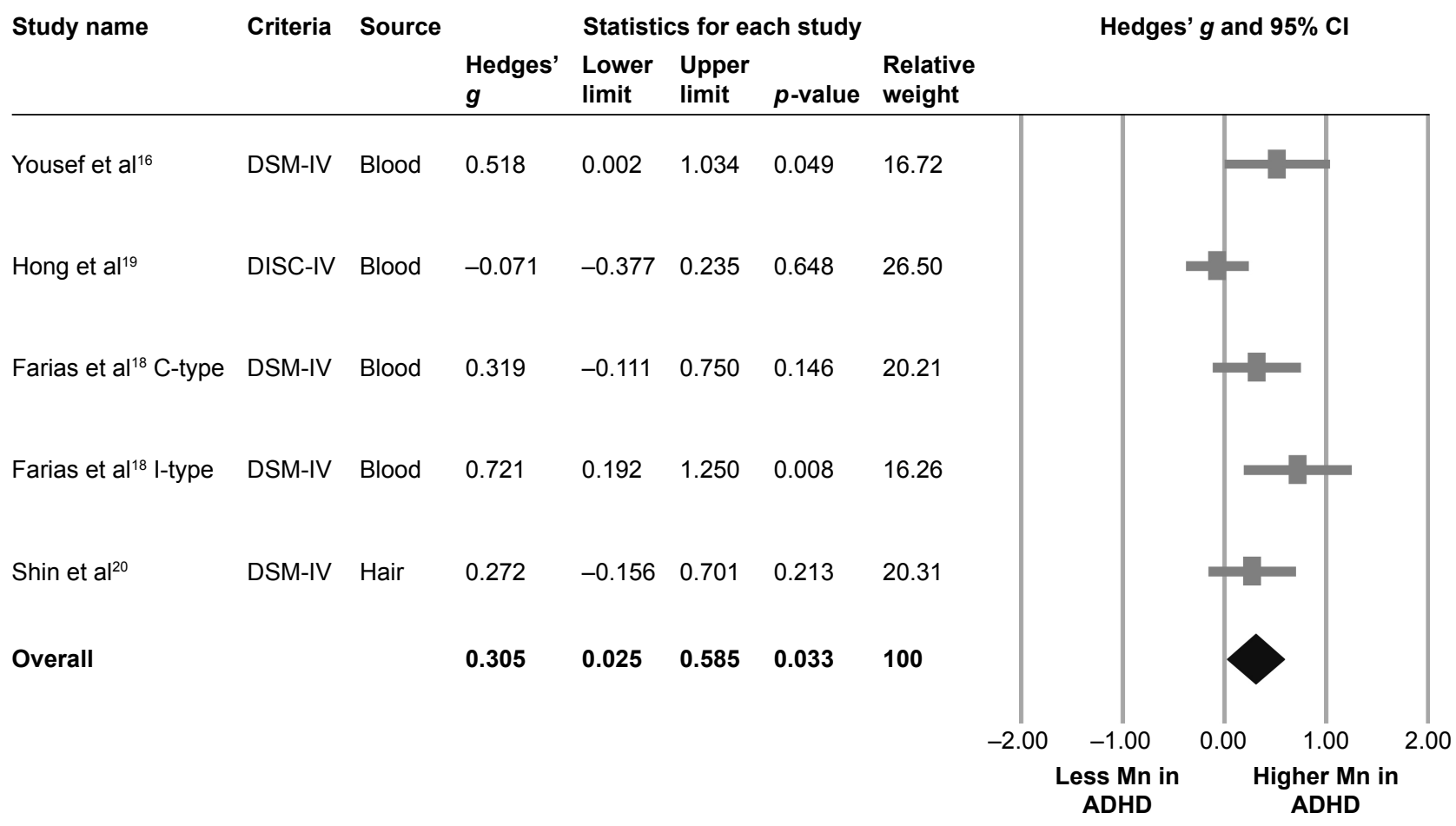

\section{B}

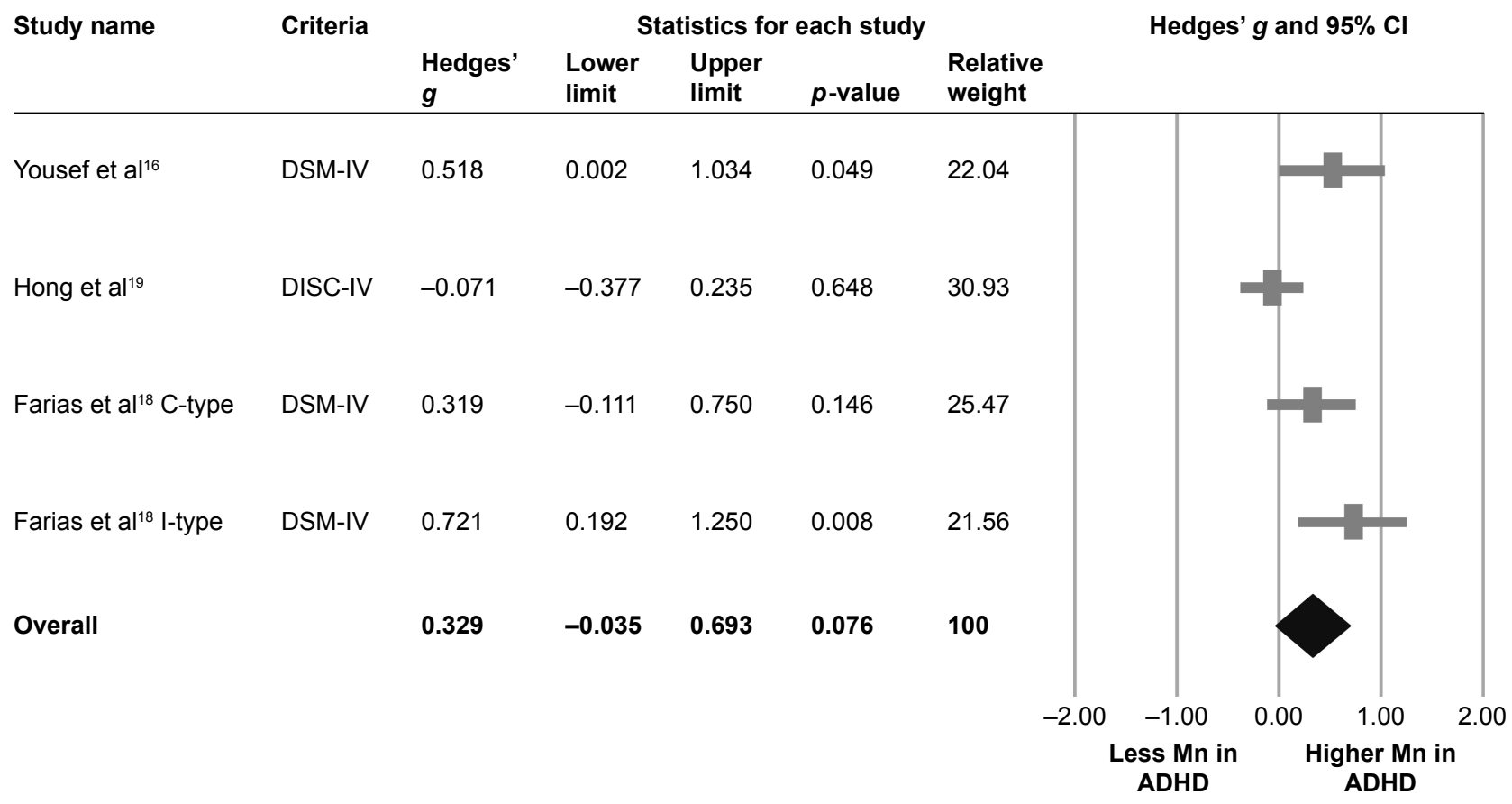

Figure 2 (Continued) 


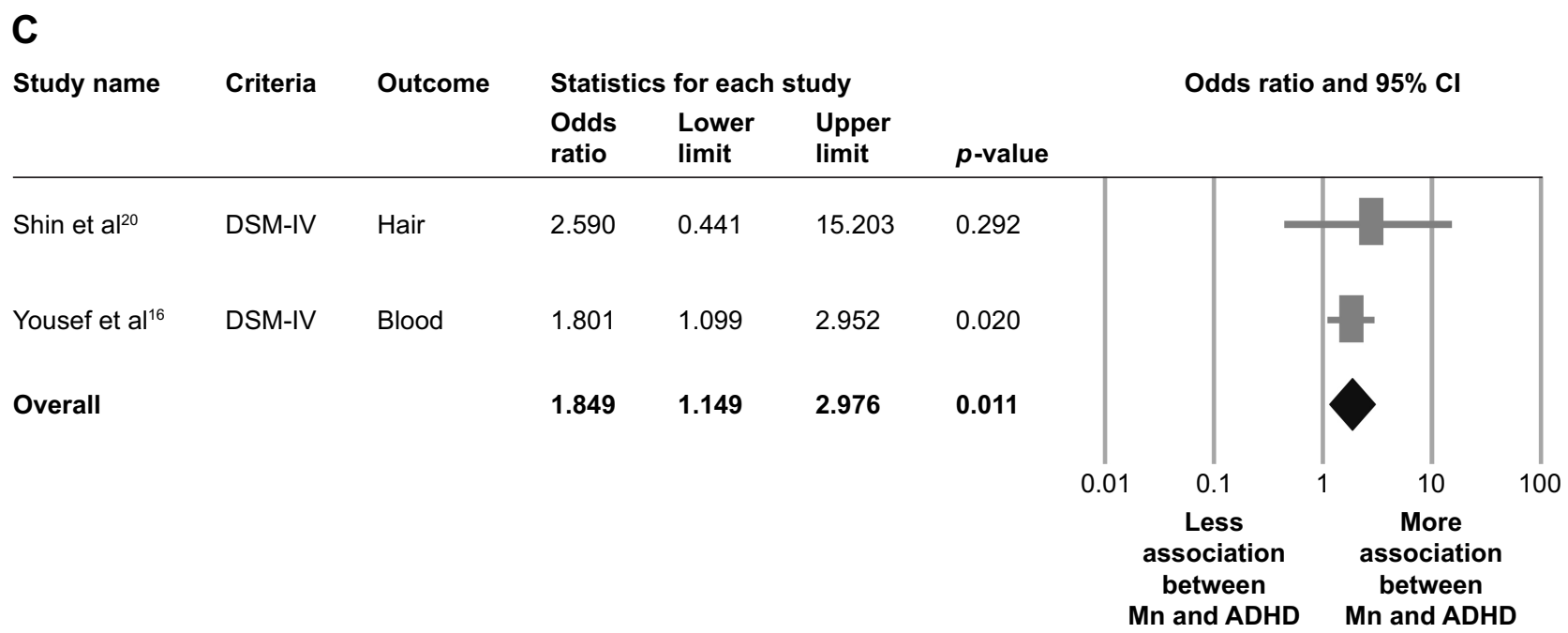

Figure 2 Meta-analysis of (A) overall manganese levels in ADHD children and controls, (B) blood manganese levels in ADHD children and controls, and (C) pooled adjusted OR for the association between manganese levels and ADHD.

Notes: (A) There were significantly higher overall manganese levels in ADHD children than those in controls (Hedges' $g=0.305,95 \% \mathrm{Cl}=0.025-0.585, p=0.033)$. (B) There were no significantly different blood manganese levels in ADHD children and controls (Hedges' $g=0.329,95 \% \mathrm{Cl}=-0.035$ to $0.693, p=0.076$ ). (C) There was a significant association between manganese levels and diagnosis of $A D H D$ as shown by pooled adjusted $O R(O R=1.849,95 \% \mathrm{Cl}=1.149-2.976, p=0.01 \mathrm{I})$.

Abbreviations: ADHD, attention-deficit/hyperactivity disorder; C-type, combined type; DISC-IV, Diagnostic Interview Schedule for Children Version IV; DSM-IV, Diagnostic and Statistical Manual of Mental Disorders, Fourth Edition; I-type, inattention type.

meta-analysis, the study by Hong et $\mathrm{al}^{19}$ used cross-sectional design, while the other three studies ${ }^{16,18,20}$ used case-control design. Moreover, the study by Hong et al ${ }^{19}$ used Diagnostic Interview Schedule for Children Version IV, while the other studies used DSM criteria. ${ }^{16,18,20}$

Although the meta-analysis was not done in the "hair" subgroup due to the availability of only one dataset, the result of this article showed that the hair manganese levels were significantly higher in ADHD children than in controls. To overcome the potential confounding effects, we performed a meta-analysis of overall adjusted ORs, which revealed a significantly positive association (adjusted $\mathrm{OR}=1.849, p=0.011$ ) between manganese levels and ADHD in children, with no significant heterogeneity.

A comparison of the two previous meta-analyses ${ }^{17,21}$ mentioning the association between manganese and ADHD and the current study is summarized in Table 2. In the two studies, the one by Rodríguez-Barranco et $\mathrm{al}^{17}$ calculated only the association between manganese exposure and IQ, and the other by Scassellati et $\mathrm{al}^{21}$ did not perform a meta-analysis for manganese exposure.
Since "dopamine hypothesis"2 is currently the most widely postulated etiologic scheme for ADHD, disrupted dopamine neurotransmission caused by manganese exposure found in several previous studies ${ }^{13,14}$ has prompted us to perform this study. Our study results also support the positive association between peripheral manganese levels and ADHD. Although there have been no studies investigating the effect of removing excessive manganese from children with ADHD, the results of our current study suggest that this treatment approach may be promising.

Because manganese levels in children (biological monitoring) are affected by environmental manganese levels, such as in soil, water, and food, it would be difficult to thoroughly investigate. Therefore, we chose blood and hair levels as an indicator of internal dose.

The current study has several limitations. First, although our main results showed higher manganese levels in children diagnosed with ADHD than those in controls, significant publication bias and heterogeneity were found. Further, the results changed to insignificant after refilling two potential missing data by using Duval and Tweedie's trim-and-fill test

Table 2 Comparison of previous and current meta-analyses

\begin{tabular}{llll}
\hline Study & Study designs included & Manganese exposure measure & Observed effect \\
\hline Scassellati et al ${ }^{2 !}$ & Case-control & N/A & N/A \\
Rodríguez-Barranco et al ${ }^{77}$ & Case-control, cohort, & Blood, cord blood, hair, placenta, \\
& cross-sectional & tooth, water & Blood, hair \\
Current study & Case-control, cross-sectional & IQ, verbal IQ & ADHD \\
\hline
\end{tabular}

Abbreviations: ADHD, attention-deficit/hyperactivity disorder; IQ, intelligence quotient; N/A, not available. 
(Hedges' $g=0.145,95 \% \mathrm{CI}=-0.140$ to 0.430 ). The publication bias may be overcome when more primary researches are published. The heterogeneity might be due to the difference in study designs. Among the four included studies, the study by Hong et a $1^{19}$ used cross-sectional design, while the other three studies ${ }^{16,18,20}$ used matched case-control design. Another source of heterogeneity may have come from the fact that Farias et a $\mathrm{l}^{18}$ had two datasets of different diagnostic criteria, which were ADHD-combined type (C-type) and ADHD-inattentive type (I-type), respectively. Further, the four included studies investigated different populations: in terms of countries, for example, their subjects were from United Arab Emirates, ${ }^{16}$ Brazil,${ }^{18}$ and Republic of Korea. ${ }^{19,20}$ Second, due to the small number of eligible studies, we had to combine the results from blood and hair samples, which could be a source of bias. Third, there were only two datasets provided with adjusted ORs, so the potential confounding factors may not have been addressed well enough. Fourth, due to difficulties in experimental designing and ethical concerns, few studies ${ }^{34,35}$ provided central nervous system (CNS) concentrations of manganese, and none of them studied the relations between CNS manganese concentrations and ADHD. Although CNS concentrations are likely to provide more accurate information for manganese neurotoxicity, only blood or hair concentrations were available for this metaanalysis. Fifth, although eligible articles studied manganese levels in blood and hair, there have been no well-validated biomarkers for manganese exposure in children. ${ }^{36}$ Moreover, hair samples are susceptible to exogenous contamination. ${ }^{36}$ Sixth, although we provided evidences of higher manganese levels in ADHD children than in controls, we could not provide further detailed information about "to what extent" the manganese levels were higher than controls. Because the peripheral samples of manganese and method of detection in each included study were different from the others, we could not directly count and pool the difference in mean manganese levels between ADHD children and controls in each study. Seventh, although two of the studies provided information about ADHD subtype, ${ }^{16,18}$ there was still limited information about the correlation between peripheral manganese levels and ADHD subtype. Therefore, we could not derive further evidence about the correlation between peripheral manganese levels and ADHD subtype. Finally, because there had been limited articles included in the current meta-analysis, the subjects distribution was as restricted as only from three countries, including United Arab Emirates, ${ }^{16}$ Brazil,${ }^{18}$ and Republic of Korea. ${ }^{19,20}$ Therefore, when clinicians apply our results into clinical practice, they should pay more attention about the applicability due to limited number of countries included and regional bias.

\section{Conclusion}

The results of this meta-analysis support higher peripheral manganese levels in children diagnosed with ADHD than those in controls. Further studies are warranted to replicate these findings, especially in other countries and in different ADHD subtypes, and to quantify the degree of the association for it to be useful in clinical settings.

\section{Disclosure}

The authors report no conflicts of interest in this work.

\section{References}

1. American Psychiatric Association. Diagnostic and Statistical Manual of Mental Disorders. 5th ed. Arlington, VA: American Psychiatric Publishing; 2013.

2. Kliegman R, Behrman RE, Nelson WE. Nelson Textbook of Pediatrics. 20th ed. Atlanta, GA: Elsevier; 2016.

3. Polanczyk G, de Lima MS, Horta BL, Biederman J, Rohde LA. The worldwide prevalence of ADHD: a systematic review and metaregression analysis. Am J Psychiatry. 2007;164(6):942-948.

4. Huang $\mathrm{S}, \mathrm{Hu} \mathrm{H}$, Sánchez BN, et al. Childhood blood lead levels and symptoms of attention deficit hyperactivity disorder (ADHD): a cross-sectional study of Mexican children. Environ Health Perspect. 2016;124(6):868-874.

5. Lam J, Lanphear BP, Bellinger D, et al. Developmental PBDE exposure and IQ/ADHD in childhood: a systematic review and meta-analysis. Environ Health Perspect. 2017;125(8):086001.

6. Cortese S. The neurobiology and genetics of attention-deficit/ hyperactivity disorder (ADHD): what every clinician should know. Eur J Paediatr Neurol. 2012;16(5):422-433.

7. Aschner M, Guilarte TR, Schneider JS, Zheng W. Manganese: recent advances in understanding its transport and neurotoxicity. Toxicol Appl Pharmacol. 2007;221(2):131-147.

8. Guilarte TR, Chen MK, McGlothan JL, et al. Nigrostriatal dopamine system dysfunction and subtle motor deficits in manganese-exposed non-human primates. Exp Neurol. 2006;202(2):381-390.

9. Grant K, Goldizen FC, Sly PD, et al. Health consequences of exposure to e-waste: a systematic review. Lancet Glob Health. 2013;1(6): e350-e361.

10. Heacock M, Kelly CB, Asante KA, et al. E-waste and harm to vulnerable populations: a growing global problem. Environ Health Perspect. 2016;124(5):550-555.

11. Klaassen CD, Casarett LJ, Doull J. Casarett and Doull's Toxicology: The Basic Science of Poisons. 8th ed. New York, NY: McGraw-Hill Education/Medical; 2013.

12. Morello M, Canini A, Mattioli P, et al. Sub-cellular localization of manganese in the basal ganglia of normal and manganese-treated rats: an electron spectroscopy imaging and electron energy-loss spectroscopy study. Neurotoxicology. 2008;29(1):60-72.

13. McDougall SA, Reichel CM, Farley CM, et al. Postnatal manganese exposure alters dopamine transporter function in adult rats: potential impact on nonassociative and associative processes. Neuroscience. 2008;154(2):848-860.

14. Chen $\mathrm{P}, \mathrm{Chen} \mathrm{Z}, \mathrm{Li} \mathrm{A}$, et al. Catalytic metalloporphyrin protects against paraquat neurotoxicity in vivo. Biomed Environ Sci. 2008;21(3): 233-238. 
15. Liu W, Huo X, Liu D, Zeng X, Zhang Y, Xu X. S100ß in heavy metalrelated child attention-deficit hyperactivity disorder in an informal e-waste recycling area. Neurotoxicology. 2014;45:185-191.

16. Yousef S, Adem A, Zoubeidi T, Kosanovic M, Mabrouk AA, Eapen V. Attention deficit hyperactivity disorder and environmental toxic metal exposure in the United Arab Emirates. J Trop Pediatr. 2011; 57(6):457-460.

17. Rodríguez-Barranco M, Lacasaña M, Aguilar-Garduño C, et al. Association of arsenic, cadmium and manganese exposure with neurodevelopment and behavioural disorders in children: a systematic review and meta-analysis. Sci Total Environ. 2013;454-455:562-577.

18. Farias AC, Cunha A, Benko CR, et al. Manganese in children with attention-deficit/hyperactivity disorder: relationship with methylphenidate exposure. J Child Adolesc Psychopharmacol. 2010;20(2):113-118.

19. Hong SB, Kim JW, Choi BS, et al. Blood manganese levels in relation to comorbid behavioral and emotional problems in children with attention-deficit/hyperactivity disorder. Psychiatry Res. 2014;220(1-2): 418-425.

20. Shin DW, Kim EJ, Lim SW, Shin YC, Oh KS, Kim EJ. Association of hair manganese level with symptoms in attention-deficit/hyperactivity disorder. Psychiatry Investig. 2015;12(1):66-72.

21. Scassellati C, Bonvicini C, Faraone SV, Gennarelli M. Biomarkers and attention-deficit/hyperactivity disorder: a systematic review and meta-analyses. J Am Acad Child Adolesc Psychiatry. 2012;51(10): 1003.e20-1019.e20.

22. Stroup DF, Berlin JA, Morton SC, et al. Meta-analysis of observational studies in epidemiology: a proposal for reporting. Meta-analysis of observational studies in epidemiology (MOOSE) group. JAMA. 2000;283(15):2008-2012.

23. Zeng X, Xu X, Boezen HM, Huo X. Children with health impairments by heavy metals in an e-waste recycling area. Chemosphere. 2016;148:408-415.

24. Grandjean P, Landrigan PJ. Neurobehavioural effects of developmental toxicity. Lancet Neurol. 2014;13(3):330-338.

25. Lucchini R, Placidi D, Cagna G, et al. Manganese and developmental neurotoxicity. Adv Neurobiol. 2017;18:13-34.
26. Anglin RE, Samaan Z, Walter SD, McDonald SD. Vitamin D deficiency and depression in adults: systematic review and meta-analysis. $\mathrm{Br} J$ Psychiatry. 2013;202:100-107.

27. Borenstein M, Hedges LV, Higgins JP, Rothstein HR. A basic introduction to fixed-effect and random-effects models for meta-analysis. Res Synth Methods. 2010;1(2):97-111.

28. Tobias A. Assessing the influence of a single study in meta-analysis. Stata Tech Bull. 1999;47:15-17.

29. Higgins JP, Thompson SG. Quantifying heterogeneity in a metaanalysis. Stat Med. 2002;21(11):1539-1558.

30. Borenstein M, Higgins JP, Hedges LV, Rothstein HR. Basics of metaanalysis: $\mathrm{I}^{2}$ is not an absolute measure of heterogeneity. Res Synth Methods. 2017;8(1):5-18.

31. Higgins JP, Green S. 10.4.3.1 Recommendations on testing for funnel plot asymmetry. In: Higgins JP, Green S, editors. Cochrane Handbook for Systematic Reviews of Interventions. 5.1.0 ed. Cochrane Library; 2011. Available from: http://handbook-5-1.cochrane.org/ chapter_10/10_4_3_1_recommendations_on_testing_for_funnel_plot_ asymmetry.htm. Accessed July 02, 2018.

32. Egger M, Davey Smith G, Schneider M, Minder C. Bias in meta-analysis detected by a simple, graphical test. BMJ. 1997;315(7109):629-634.

33. Duval S, Tweedie R. Trim and fill: a simple funnel-plot-based method of testing and adjusting for publication bias in meta-analysis. Biometrics. 2000;56(2):455-463.

34. Franěk T, Kotaška K, Průša R. Manganese and selenium concentrations in cerebrospinal fluid of seriously ill children. J Clin Lab Anal. 2017;31(6):e22122.

35. Romarís EM, Cervantes II, López JM, Marcén JF. Concentration of calcium and magnesium and trace elements (zinc, copper, iron and manganese) in cerebrospinal fluid: a try of a pathophysiological classification. J Trace Elem Med Biol. 2011;25(Suppl 1):S45-S49.

36. Eastman RR, Jursa TP, Benedetti C, Lucchini RG, Smith DR. Hair as a biomarker of environmental manganese exposure. Environ Sci Technol. 2013;47(3):1629-1637. 


\section{Supplementary materials}

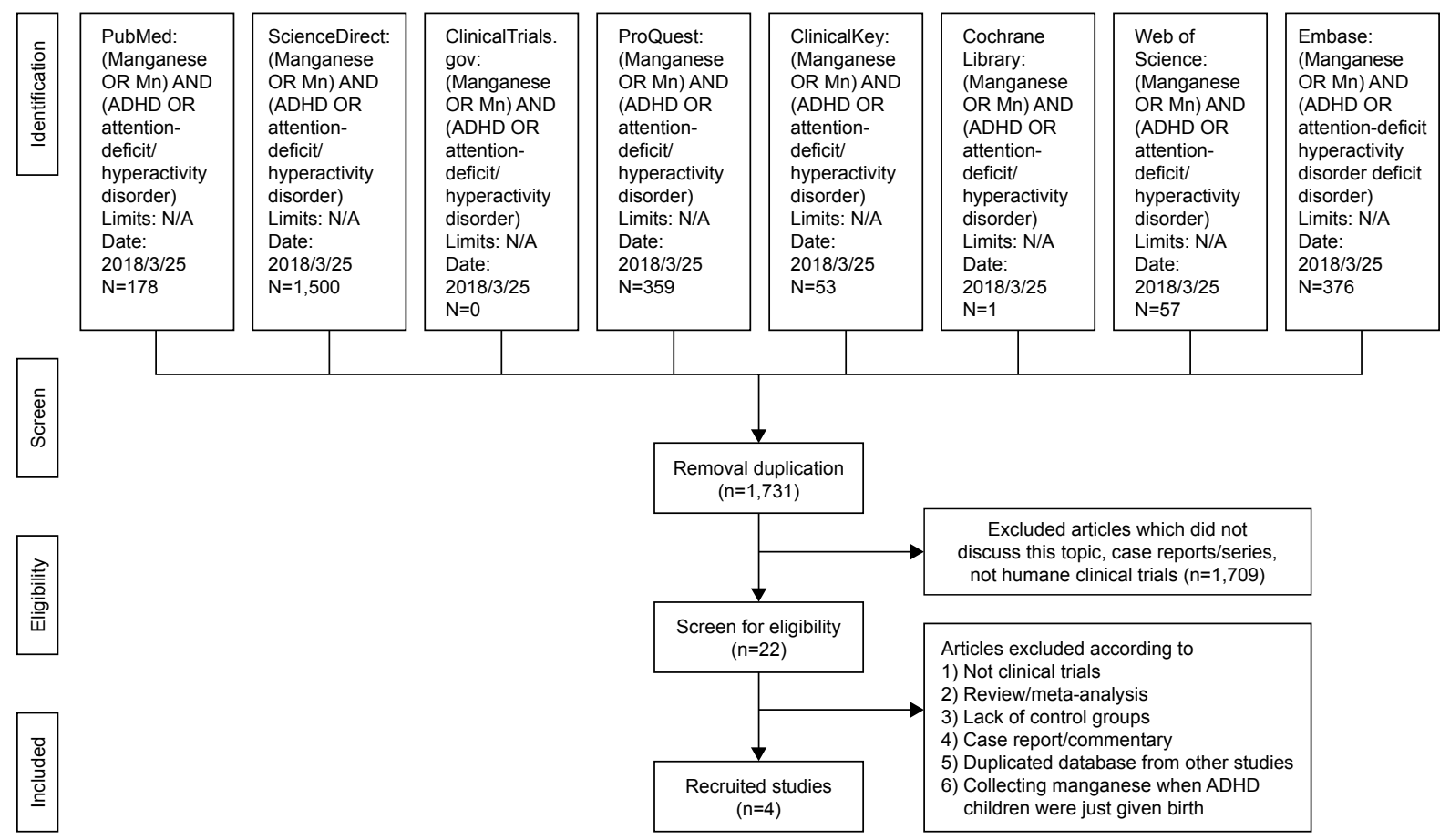

Figure SI Whole flowchart of the meta-analysis.

Abbreviations: ADHD, attention-deficit/hyperactivity disorder; N/A, not available.

Table SI Results of Newcastle-Ottawa Scale scores of recruited studies

\begin{tabular}{|c|c|c|c|c|c|c|c|c|c|}
\hline \multirow[t]{2}{*}{ Study } & \multirow[t]{2}{*}{ Criteria } & \multirow[t]{2}{*}{ Source } & \multirow[t]{2}{*}{ Sample method } & \multicolumn{5}{|c|}{ Newcastle-Ottawa Scale } & \multirow[t]{2}{*}{ Country } \\
\hline & & & & Selection & Performance & Detection & Information & Total & \\
\hline Shin et al' & DSM-IV & Hair & $\begin{array}{l}\text { Inductively coupled } \\
\text { plasma mass spectrometry }\end{array}$ & $\mathrm{I}$ & 5 & 4 & 5 & 15 & Republic of Korea \\
\hline Hong et $\mathrm{al}^{2}$ & DISC-IV & Blood & $\begin{array}{l}\text { Graphite furnace atomic } \\
\text { absorption spectrometry }\end{array}$ & 3 & 5 & 5 & 4 & 17 & Republic of Korea \\
\hline Yousef et $\mathrm{al}^{3}$ & DSM-IV & Blood & N/A & 2 & 5 & 4 & 4 & 15 & United Arab Emirates \\
\hline Farias et $\mathrm{al}^{4}$ & DSM-IV & Blood & $\begin{array}{l}\text { Graphite furnace atomic } \\
\text { absorption spectrometry }\end{array}$ & 2 & 5 & 4 & 4 & 15 & Brazil \\
\hline
\end{tabular}

Abbreviations: DSM-IV, Diagnostic and Statistical Manual of Mental Disorders, Fourth Edition; DISC-IV, Diagnostic Interview Schedule for Children Version IV; N/A, not available. 
Table S2 Excluded studies and reasons

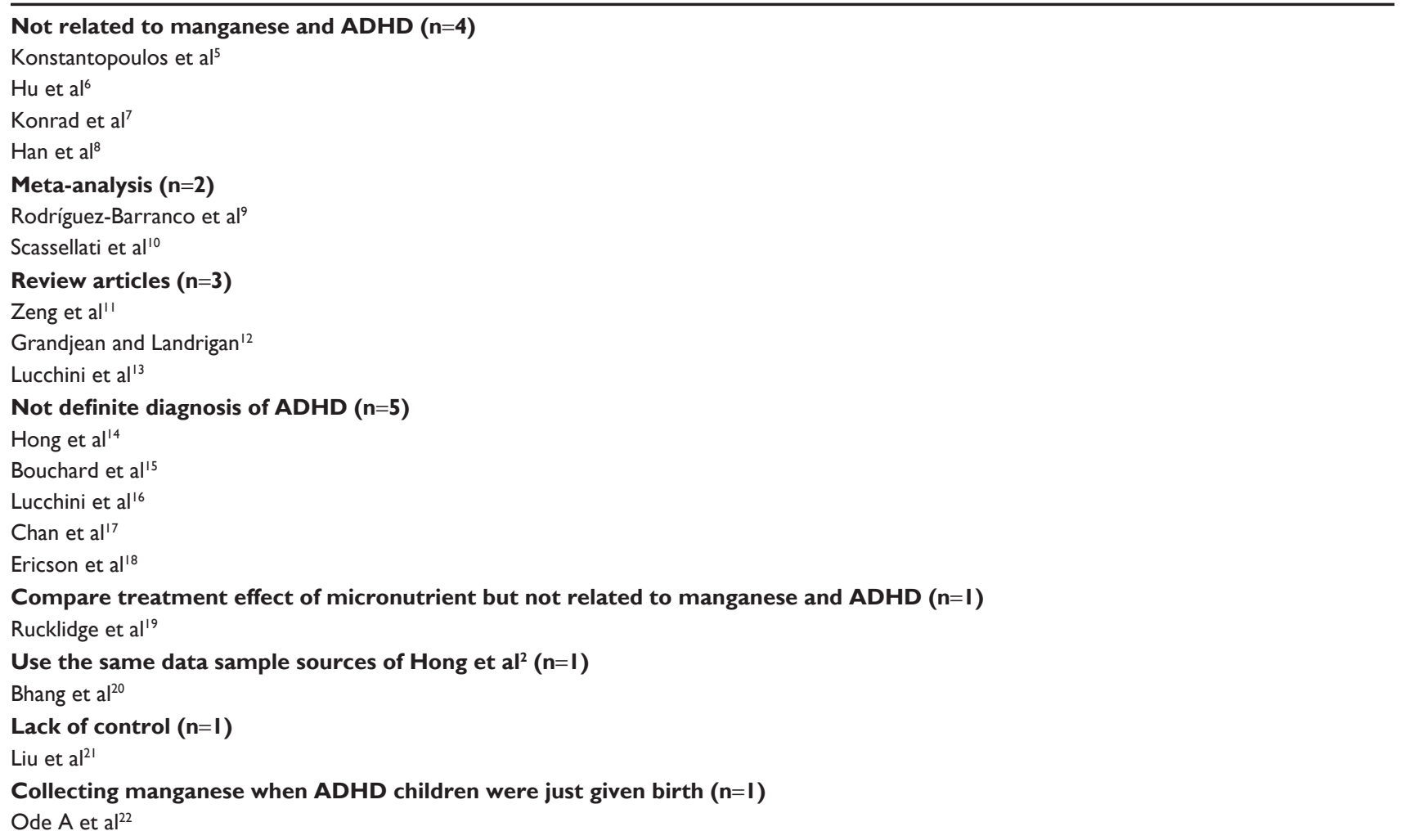

Abbreviation: ADHD, attention-deficit/hyperactivity disorder.

\section{References}

1. Shin DW, Kim EJ, Lim SW, Shin YC, Oh KS, Kim EJ. Association of hair manganese level with symptoms in attention-deficit/hyperactivity disorder. Psychiatry Investig. 2015;12(1):66-72.

2. Hong SB, Kim JW, Choi BS, et al. Blood manganese levels in relation to comorbid behavioral and emotional problems in children with attention-deficit/hyperactivity disorder. Psychiatry Res. 2014; 220(1-2):418-425.

3. Yousef S, Adem A, Zoubeidi T, Kosanovic M, Mabrouk AA, Eapen V. Attention deficit hyperactivity disorder and environmental toxic metal exposure in the United Arab Emirates. J Trop Pediatr. 2011; 57(6):457-460.

4. Farias AC, Cunha A, Benko CR, et al. Manganese in children with attention-deficit/hyperactivity disorder: relationship with methylphenidate exposure. J Child Adolesc Psychopharmacol. 2010;20(2):113-118.

5. Konstantopoulos K, Vogazianos P, Thodi C, Nikopoulou-Smyrni P. A normative study of the Children's Color Trails Test (CCTT) in the Cypriot population. Child Neuropsychol. 2015;21(6):751-758.

6. Hu D, Wang YX, Chen WJ, et al. Associations of phthalates exposure with attention deficits hyperactivity disorder: a case-control study among Chinese children. Environ Pollut. 2017;229:375-385.

7. Konrad K, Gauggel S, Schurek J. Catecholamine functioning in children with traumatic brain injuries and children with attention-deficit/hyperactivity disorder. Brain Res Cogn Brain Res. 2003;16(3):425-433.

8. Han JY, Kwon HJ, Ha M, et al. The effects of prenatal exposure to alcohol and environmental tobacco smoke on risk for ADHD: a large population-based study. Psychiatry Res. 2015;225(1-2): $164-168$.

9. Rodríguez-Barranco M, Lacasaña M, Aguilar-Garduño C, et al. Association of arsenic, cadmium and manganese exposure with neurodevelopment and behavioural disorders in children: a systematic review and meta-analysis. Sci Total Environ. 2013;454-455:562-577.
10. Scassellati C, Bonvicini C, Faraone SV, Gennarelli M. Biomarkers and attention-deficit/hyperactivity disorder: a systematic review and meta-analyses. J Am Acad Child Adolesc Psychiatry. 2012;51(10): 1003-1019.e20.

11. Zeng X, Xu X, Boezen HM, Huo X. Children with health impairments by heavy metals in an e-waste recycling area. Chemosphere. 2016;148: $408-415$.

12. Grandjean P, Landrigan PJ. Neurobehavioural effects of developmental toxicity. Lancet Neurol. 2014;13(3):330-338.

13. Lucchini R, Placidi D, Cagna G, et al. Manganese and developmental neurotoxicity. Adv Neurobiol. 2017;18:13-34.

14. Hong SB, Im MH, Kim JW, et al. Environmental lead exposure and attention deficit/hyperactivity disorder symptom domains in a community sample of South Korean school-age children. Environ Health Perspect. 2015;123(3):271-276.

15. Bouchard M, Laforest F, Vandelac L, Bellinger D, Mergler D. Hair manganese and hyperactive behaviors: pilot study of school-age children exposed through tap water. Environ Health Perspect. 2007; 115(1):122-127.

16. Lucchini RG, Zoni S, Guazzetti S, et al. Inverse association of intellectual function with very low blood lead but not with manganese exposure in Italian adolescents. Environ Res. 2012;118:65-71.

17. Chan TJ, Gutierrez C, Ogunseitan OA. Metallic burden of deciduous teeth and childhood behavioral deficits. Int J Environ Res Public Health. 2015;12(6):6771-6787.

18. Ericson JE, Crinella FM, Clarke-Stewart KA, et al. Prenatal manganese levels linked to childhood behavioral disinhibition. Neurotoxicol Teratol. 2007;29(2):181-187.

19. Rucklidge J, Johnstone J, Harrison R, Boggis A. Micronutrients reduce stress and anxiety in adults with attention-deficit/hyperactivity disorder following a 7.1 earthquake. Psychiatry Res. 2011;189(2):281-287. 
20. Bhang SY, Cho SC, Kim JW, et al. Relationship between blood manganese levels and children's attention, cognition, behavior, and academic performance-a nationwide cross-sectional study. Environ Res. 2013;126: 9-16.

21. Liu W, Huo X, Liu D, Zeng X, Zhang Y, Xu X. S100ß in heavy metalrelated child attention-deficit hyperactivity disorder in an informal e-waste recycling area. Neurotoxicology. 2014;45:185-191.
22. Ode A, Rylander L, Gustafsson P, et al. Manganese and selenium concentrations in umbilical cord serum and attention deficit hyperactivity disorder in childhood. Environ Res. 2015;137:373-381.

\section{Publish your work in this journal}

Neuropsychiatric Disease and Treatment is an international, peerreviewed journal of clinical therapeutics and pharmacology focusing on concise rapid reporting of clinical or pre-clinical studies on a range of neuropsychiatric and neurological disorders. This journal is indexed on PubMed Central, the 'PsycINFO' database and CAS, and is the official journal of The International Neuropsychiatric Association (INA). The manuscript management system is completely online and includes a very quick and fair peer-review system, which is all easy to use. Visit http://www.dovepress.com/testimonials.php to read real quotes from published authors.

\footnotetext{
Submit your manuscript here: http://www.dovepress.com/neuropsychiatric-disease-and-treatment-journal
} 